\title{
Female predisposition to cranial neural tube defects is not because of a difference between the sexes in the rate of embryonic growth or development during neurulation
}

\author{
Frances A Brook, J Peter Estibeiro, Andrew J Copp
}

\begin{abstract}
The susceptibility of females to anencephaly is well established and has been suggested to result from a slower rate of growth and development of female embryos during cranial neurulation. We have tested this hypothesis by measuring the rates of growth and development, both in utero and in vitro, of male and female embryos of the curly tail (ct) mutant mouse strain, in which cranial neural tube defects occur primarily in females. Embryonic growth was assessed by increase in protein content, while development progression was judged from increase in somite number and morphological score. Embryos were sexed by use of the polymerase chain reaction to amplify a DNA sequence specific to the $Y$ chromosome, and by sex chromatin analysis. We find that, during neurulation (between 8.5 and 10.5 days of gestation), males are advanced in growth and development relative to their female litter mates, but that the rates of growth and development do not differ between the sexes during this period. We conclude that rate of embryonic growth and development is unlikely to determine susceptibility to cranial neural tube defects. It seems more likely that male and female embryos differ in some specific aspect(s) of the neurulation process that increases the susceptibility of females to development of anencephaly.
\end{abstract}

\section{(f Med Genet 1994;31:383-387)}

Neural tube defects (NTD) are among the commonest of human congenital malformations which, if they occur at the cranial end of the body axis, result in anencephaly or, if they occur more caudally, give rise to spina bifida. The sex distribution of NTD has been well documented. Among fetuses and newborns with anencephaly (but not with spina bifida), there is a marked preponderance of females; the male/female ratio varies from 0.33 to $0 \cdot 66 .^{1-3}$ The excess of females is more marked the greater the overall incidence of anencephaly. ${ }^{4}$

Several hypotheses have been proposed to account for the greater susceptibility of females to anencephaly. ${ }^{15-7}$ In particular, an idea that has gained considerable credence is that female embryos have a slower rate of growth than males; according to this hypothesis the female excess among anencephalics is believed to be the result of the longer time period occupied by neurulation in the female and the correspondingly greater opportunity for teratogenic perturbation. . $^{8-10}$ Among normal human fetuses, males are larger than females by the end of the first trimester. ${ }^{11}$ However, little is known about the relative growth rates of male and female embryos during the critical time period when the neural tube is closing, between the third and fourth weeks of gestation. Such information is difficult to obtain at this early stage of human pregnancy and it is here that an animal model is of value.

The curly tail $(c t)$ mouse is a mutant strain with a predisposition for neurulation defects that closely resemble NTD in humans. ${ }^{12}{ }^{13} \mathrm{Ap}-$ proximately 40 to $60 \%$ of embryos homozygous for the mutation develop spinal NTD that include lumbosacral spina bifida and tail flexion defects. The incidence of exencephaly (the developmental progenitor of anencephaly) varies from $1 \%$ to $9 \%$, but always shows a pronounced female excess: the male/female ratio is about $0 \cdot 25 .^{1314}$ It has been shown that at the time when neural tube closure starts, male $c t$ embryos are developmentally advanced relative to females. ${ }^{10}$ However, measurements made at a single time point provide no information about the rate of development. The greater size of male embryos may be because of a more rapid rate of growth and development during neurulation in males than females; alternatively, the rates of growth and development during neurulation may be similar and the larger size of the males may be the result of earlier developmental events.

In the present study, we have measured several parameters relating to the development and growth of male and female $c t$ embryos throughout the period of neural tube closure. Our results indicate that although development is more advanced in males than females, there is no difference between male and female embryos in their rates of development and growth at this time, contrary to the widely held belief.

Materials and methods

DEVELOPMENT AND GROWTH IN UTERO Collection of embryos

Curly tail mice were maintained on a light/ dark cycle with the dark period from $1.00 \mathrm{am}$ 
to $9.00 \mathrm{am}$. Animals were paired for mating at 7.30 am and females were checked for copulation plugs three to five hours later. Ovulation is believed to occur around the middle of the dark period and the animals mate shortly after pairing (FAB and AJC, unpublished observations); therefore the gestational age of the embryos was calculated from the time of pairing.

Embryos were collected between 8.5 and 10.5 days of gestation (day of finding a plug was designated 0.5 days of gestation). The embryos were separated from the decidua and trophoblast and the yolk sac circulation was scored (as part of the Brown-Fabro scoring system, see below). The extra-embryonic membranes were then removed, rinsed in phosphate buffered saline, $\mathrm{pH} 7 \cdot 4$, and stored frozen for subsequent sexing. Embryonic development was assessed by counting the number of somite pairs and by determining the morphological score. ${ }^{15}$ Embryonic growth was determined by measuring the total protein content of each embryo. ${ }^{16}$

\section{Sex determination}

DNA was extracted from the yolk sac and amnion as described previously. ${ }^{17} \mathrm{~A}$ sequence specific for the $\mathrm{Y}$ chromosome, within the Zfy locus, was amplified by the polymerase chain reaction $(\mathrm{PCR})$, using oligonucleotide primers and reaction conditions as described previously. ${ }^{18}$ The products were separated by electrophoresis on $1.5 \%$ agarose gels and bands were visualised under UV by staining with ethidium bromide: A strongly fluorescing male specific band was seen (arrow b, fig 1). A second, much fainter band (possibly amplified from the related $\mathrm{Zfx}$ sequence) was detected in DNA from all embryos (arrow a, fig 1); this second band served as an internal loading control. All samples were sexed in duplicate.

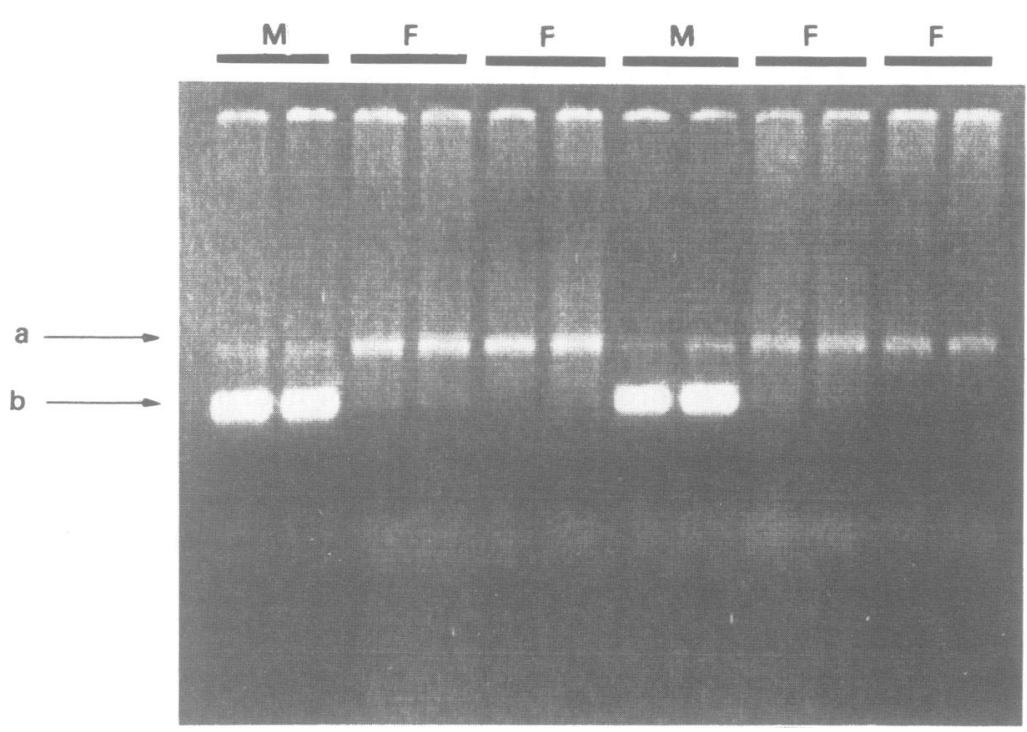

Figure $1 Y$ chromosome specific sequence (arrow b) amplified by PCR and visualised on a $1.5 \%$ agarose gel stained with ethidium bromide. A second, much fainter band (arrow a) is present in DNA from all embryos; this serves as a loading control. Each embryo is analysed in duplicate in adjacent lanes. On this gel, two males and four females are identified.
DEVELOPMENT AND GROWTH IN VITRO

Curly tail embryos were explanted from the uterus at 8.5 days of gestation, into alpha modification of Eagle's medium containing $10 \%$ fetal calf serum. The number of somite pairs was counted and crown-rump length was measured using an eyepiece graticule fitted on a Wild M7S dissecting microscope. Individual embryos were identified by tagging with pieces of suture thread as described previously. ${ }^{19} \mathrm{Em}$ bryos were cultured in whole rat serum for 48 hours by a standard method..$^{2021}$

After culture, the embryos were dissected free of their extra-embryonic membranes and the number of somite pairs was counted. Protein content could not be used as a measure of growth in this experiment, as the intention was to make multiple measurements on individual embryos. Crown-rump length was measured instead. Protein content and crown-rump length are both known to correlate with gestational age in developing rodent embryos. ${ }^{15}$ The in vitro experiment was performed before the establishment of the PCR sexing method, so the sex of the embryos was determined by sex chromatin analysis. The amnions were fixed and processed as described previously ${ }^{22}$ and the slide preparations were stained with lactoacetic orcein. Each slide was scored "blind" for the presence of sex chromatin by two independent observers.

\section{ANALYSIS OF DATA}

Within each litter of embryos in utero, means were calculated separately for males and females for each of the following parameters: somite number, morphological score, and protein content. Only embryos lying within the $95 \%$ confidence intervals were included when calculating the means; any runtish embryos that fell outside these limits were excluded. All analyses were carried out using these mean values. Differences between male and female embryos were examined by the method of paired comparisons. ${ }^{23}$ Multiple regression analysis, using the statistical software package Minitab, was carried out to examine the effects of sex, gestational age, and litter size, and their interactions, upon each of the three parameters studied. Use of the whole embryo culture method enabled us to determine the rate of development and growth of individual embryos during a 48 hour period in vitro. Mean somite number and crown-rump length at the start and end of culture, and the rate of increase of these parameters during culture, were compared between male and female embryos using Student's $t$ test, computed using the Minitab statistical software package.

\section{Results}

RATE OF DEVELOPMENT AND GROWTH IN UTERO A total of 163 embryos were obtained from 20 litters, giving an average litter size of 8.2 (SE $0 \cdot 3$ ). Fifty five percent of the embryos were male; the mean number of males per litter was 4.5 (SE 0.4) and the mean number of females was 3.7 (SE $0 \cdot 3)$. This difference was not 

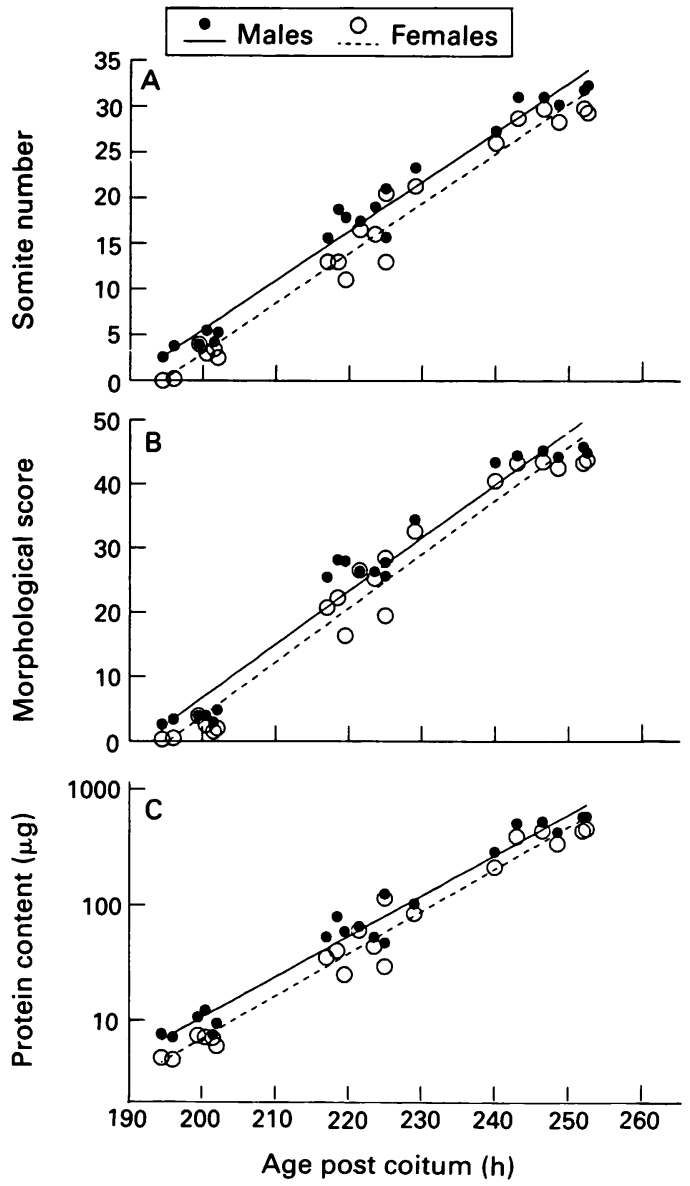

Figure 2 Rate of development, as assessed by $(A)$ somite number and $(B)$ morphological score, and rate of growth, as assessed by (C) protein content (plotted on a logarithmic scale), in male and female ct embryos. For each parameter, mean value per litter is plotted against gestational age and separate regression lines are drawn through the male (solid circles, continuous lines) and female (hollow circles, dashed lines) data. There is no significant difference between the sexes in the rate of increase of somite number, morphological score, or protein content as shown by the closely similar gradients of the regression lines. Males are consistently ahead of their female litter mates in all three parameters, as shown by the higher value of the $y$ axis intercept for the male regression lines.

significant $(p>0.05)$. Six of the embryos, all from separate litters, fell outside the $95 \%$ confidence limits and were excluded from the analysis. Four were male, two were female, and all were substantially smaller than their litter mates. Their exclusion, as runts, does not bias the following results.

Developmental stage of $c t$ embryos from 8.5 to 10.5 days of gestation was assessed from somite counts and by staging embryos according to the Brown-Fabro scoring system. There was a significant difference in the developmental stage reached by males and females in

Somite number and crown-rump length of curly tail embryos during 48 hour culture

\begin{tabular}{|c|c|c|c|c|c|c|}
\hline & \multicolumn{2}{|c|}{ Before culture } & \multicolumn{2}{|c|}{ After culture } & \multicolumn{2}{|c|}{ Gain during culture } \\
\hline & Somites* & $C R$ length* & Somites & CR length & Somites & CR length \\
\hline $\begin{array}{l}\begin{array}{l}\text { Males } \\
(n=19)\end{array} \\
\text { Females } \\
(n=18) \\
p \text { value } \dagger\end{array}$ & $\begin{array}{c}4.9 \\
(0.5) \\
3 \cdot 2 \\
(0.5) \\
0.01\end{array}$ & $\begin{array}{c}0.72 \\
(0.05) \\
0.61 \\
(0.03) \\
0.05\end{array}$ & $\begin{array}{c}29 \cdot 4 \\
(0.5) \\
27 \cdot 6 \\
(0.6) \\
0.03\end{array}$ & $\begin{array}{c}2.29 \\
(0.07) \\
2 \cdot 11 \\
(0.07) \\
0.07\end{array}$ & $\begin{array}{c}24 \cdot 5 \\
(0 \cdot 2) \\
24 \cdot 4 \\
(0 \cdot 3) \\
0 \cdot 83\end{array}$ & $\begin{array}{c}1.57 \\
(0.04) \\
1.50 \\
(0.04) \\
0.27\end{array}$ \\
\hline
\end{tabular}

* Values are means (SE). Crown-rump (CR) length values are in $\mathrm{mm}$.

t $\mathrm{p}$ values obtained from Student's $t$ test comparisons between male and female data. the same litter $(p<0.001)$. Out of the 20 litters studied, the mean somite number of the males was greater than that of their female sibs in 17 cases and the mean morphological score of the males was greater in 19 cases. Both developmental parameters increased linearly with time between 8.5 and 10.5 days of gestation (fig 2A,B). Multiple regression analysis showed a significant effect of sex upon each parameter $(\mathrm{p}<0.001$ and $\mathrm{p}<0.05$ respectively) as indicated by the difference in $y$ axis intercepts of the regression lines. On the other hand, the rate of increase of somite number and morphological score was not significantly different between males and females $(p>0.05)$, as shown by the closely similar gradients of the regression lines (fig 2A,B). Litter size did not influence the difference in developmental advancement between males and females.

Growth of the embryos was measured by determining total protein content. In all 20 litters, the mean protein content of the male embryos was greater than that of the females $(\mathrm{p}<0.002)$. However, the rate at which protein content increased showed no difference between males and females ( $p>0.05$, fig 2C). Litter size had no effect upon the difference in growth advancement between males and females.

\section{DEVELOPMENT AND GROWTH IN VITRO}

Development and growth of individual embryos undergoing neural tube closure was followed in culture. Developmental stage was assessed by counting the somite pairs, and growth by measuring crown-rump length. Embryos gained approximately 24 somites over the 48 hour culture period (table) which is close to the expected value of one somite every two hours for this period of gestation in utero. ${ }^{15}$ This suggests that the rate of embryonic development in vitro was closely similar to that in utero.

When culture started, the mean somite number and crown-rump length of the male embryos were both greater than the corresponding values for females $(p=0.01, p=0.05$ respectively, table). At the end of the culture period, male embryos were still more advanced and larger than the females. However, the increase in the number of somites and in crown-rump length during the culture period was not significantly different between males and females $(p>0.05)$, indicating that the rates of development and growth were similar during neurulation in vitro (table).

\section{Discussion}

We have studied a mutant mouse strain with genetic predisposition to NTD, in which cranial defects show a strong female preponderance, as in humans. In this strain, we find that the overall rates of growth and development do not differ between the sexes during the embryonic process of neurulation. This conclusion is based on two independent experiments: in the first, $c t$ embryos developing in utero were sampled at different times 
during neurulation and, in the second, individual embryos were followed through 48 hours of development in vitro. It seems very unlikely, therefore, that the overall rate of growth or development at the time of cranial neural tube closure determines susceptibility to NTD, as was suggested previously. ${ }^{8-10}$

We have confirmed the findings of others that male embryos are advanced in growth and development relative to females at the time of neurulation. In view of our findings on rates of growth and development, it is clear that the advancement of males must originate earlier in development. Indeed, males are more advanced than females at preimplantation stages in mice $^{2425}$ and cattle. ${ }^{2627}$ Since this sex difference appears before $\mathrm{X}$ chromosome inactivation is complete, ${ }^{28}$ it is possible that possession of two functioning $\mathrm{X}$ chromosomes may retard embryo development. Thornhill and Burgoyne ${ }^{29}$ have shown that a paternally derived X chromosome can delay development in mice. Additionally, the presence of the $Y$ chromosome can enhance embryo development. In preimplantation embryos of $\mathrm{CDI}$ and MFI mice, the $\mathrm{Y}$ chromosome is responsible for accelerating development in the male. ${ }^{30}$

What, then, is the reason for the female predisposition to cranial NTD? We will consider four possibilities.

\section{ARE AFFECTED MALES LOST SELECTIVELY} DURING GESTATION?

The female preponderance among anencephalics could arise from the selective loss of affected males during gestation. There is some evidence that this mechanism may be responsible for the excess of females among genetically normal mouse embryos in which exencephaly was induced by hyperthermia. ${ }^{31}$ In the $c t$ mutant, however, there is no suggestion that male embryos with cranial NTD are eliminated: the overall incidence of resorptions is not higher than in other strains of mice ${ }^{13}$ and the overall sex ratio does not depart significantly from 1 . Moreover, analysis of $c t$ embryos at 11.5 and 12.5 days of gestation, only two to three days after the normal time of cranial neurulation, shows that a strong female preponderance is already established among exencephalics. ${ }^{14}$ Female embryos must, therefore, be more susceptible to the factors responsible for cranial NTD.

\section{DO FEMALES UNDERGO NEURULATION AT A} MORE IMMATURE STAGE THAN MALES?

The relative advancement of males could indicate that females pass through the various events of neurulation at a more immature stage (that is, with fewer somites). For instance, if neural tube closure occurs at the same chronological age in both sexes, the process in females would happen at a less advanced stage when there are fewer cells in the neuroepithelium and mesoderm. This might present problems in achieving normal raising and fusion of the neural folds. ${ }^{3}$ This idea is not supported, however, by our preliminary finding (Brook and
Copp, unpublished data) that cranial neural tube closure is initiated, at the midbrain/forebrain boundary, at the 9 to 12 somite stage in both sexes. Thus, cranial neurulation does not appear to be occurring at a lower cell number in female embryos than in males.

IS THERE A MATERNAL, TIME DEPENDENT CONSTRAINT ON NEURULATION?

It is possible that there is a "preferred time" in pregnancy for cranial neurulation to occur, as dictated by the maternal environment. This might favour males which undergo neurulation earlier, in chronological terms, than females. Although such a hypothesis cannot be ruled out, it seems unlikely in view of the ability of mouse and rat embryos to initiate and complete cranial neurulation normally in isolation from the maternal environment, in whole embryo culture. ${ }^{2032}$

DO MALES AND FEMALES DIFFER IN A SPECIFIC ASPECT OF THE CRANIAL NEURULATION PROCESS?

This hypothesis suggests that, while there are no overall differences between the sexes that can account for the susceptibility of females to cranial NTD, there may be subtle differences in the process of raising and fusion of the neural folds. Our preliminary studies with the $c t$ mutant (Brook and Copp, unpublished data) suggest that there is an impairment to closure of the neural folds specifically in the lower midbrain and hindbrain of females that delays neurulation in this region.

Do our findings have relevance for an understanding of human NTD? It is difficult to confirm our findings in humans directly owing to the inaccessibility of human embryos during neurulation. Nevertheless, the $c t$ mutant is considered to show a strong similarity to human NTD in several respects. Apart from the similarity in predisposition of females to cranial NTD, $c t$ mice with open neural tubes also exhibit raised levels of amniotic fluid $\alpha$ fetoprotein ${ }^{33}$ and an association with hydrocephalus and polyhydramnios. ${ }^{13}$ There is also evidence of polygenic inheritance, as probably occurs in the aetiology of human NTD. Expression of the $c t$ mutation is modified by other genes that are polymorphic between inbred mouse strains. ${ }^{1334}$ It seems likely, therefore, that the $c t$ mutant may be a good model for at least some types of human NTD and that our findings on the developmental basis of female susceptibility to cranial defects may have general relevance.

We thank John Crolla for valuable assistance with the sex chromatin preparations and Matthew Eagle for statistical advice. The early stages of this work were performed at the Paediatric Research Unit, Guy's Hospital Medical School, London and were supported by a grant from Action Research The majorted by the Imperial Cancer Research Fund and as part of a Multicenter Agreement for studying Neural Tube Defects in Mutant Mice funded by the National Institu for Child Health and Human Development, NIH, USA, through Cooperative
Agreement HD28882-01.

1 Rogers SC, Morris $M$. Anencephalus: a changing sex ratio. Br 7 Prev Soc Med 1973;27:81-4. 
2 Carter CO. Clues to the aetiology of neural tube malformations. Dev Med Child Neurol 1974;16(suppl 32):3-15.

3 Seller MJ, Neural tube defects and sex ratios. Am $\mathcal{F}$ Med Genet 1987;26:699-707.

4 James WH. The sex ratio in anencephaly. $f$ Med Genet 1979;16:129-33.

5 Janerich DT. Female excess in anencephaly and spina bifida: possible gestational influences. $A m$ f Epidemiol bifida: possible

6 Burn J, Gibbens D. May spina bifida result from an Xlinked defect in a selective abortion mechanism? $\mathcal{F} \mathrm{Med}$ Genet 1979;16:210-14.

7 James WH. A possible mechanism underlying the sex selectivity of neural tube defect. $₹$ Med Genet 1985;22:319.

8 Renwick JH. Hypothesis. Anencephaly and spina bifida are usually preventable by avoidance of a specific but unidentified substance present in

9 Golding J. Evidence for an environmental aetiology for anencephalus. In: Persaud TVN, ed. Advances in the study of birth defects. Vol 7. New York: Alan R Liss,

10 Seller MJ, Perkins-Cole KJ. Sex difference in mouse embryonic development at neurulation. $\mathcal{F}$ Reprod Ferti 1987;79:159-61.

11 Pedersen JF. Ultrasound evidence of sexual difference in fetal size in first trimester. $B M \mathcal{F}$ 1980;281:1253.

12 Gruneberg $H$. Genetical studies on the skeleton of the mouse. VIII. Curly tail. $\mathcal{F}$ Genet 1954;52:52-67.

13 Embury S, Seller MJ, Adinolfi M, Polani PE. Neural tube defects in curly-tail mice. I. Incidence and expression. Proc $R$ Soc Lond B 1979;206:85-94.

14 Copp AJ, Brook FA. Does lumbosacral spina bifida arise by failure of neural folding or by defective canalisation? Med Genet 1989;26:160-6.

15 Brown NA, Fabro S. Quantitation of rat embryonic development in vitro: a morphological scoring system. Teratology 1981;24:65-78.

16 Lowry OH, Rosebrough NJ, Farr AL, Randall RJ. Protein measurement with the Folin phenol reagent. $7 \mathrm{Biol}$ Chem 1951;193:265-75.

17 Estibeiro JP, Copp AJ, Cockroft DL, Brown NA, Clarke DO. Extraction of macromolecules from embryonic material. In: Copp AJ, Cockroft DL, eds. Postimplantation mammalian embryos: a practical approach. Oxford: IRL Press, 1990:173-204.

18 Nagamine CM, Chan K, Kozak CA, Lau Y. Chromosome mapping and expression of a putative testis-determining gene in mouse. Science 1989;243:80-3.
19 Copp AJ. Studying developmental mechanisms in intact embryos. In: Copp AJ, Cockroft DL, eds. Postimplantation mammalian embryos: a practical approach. Oxford: IRL Press, 1990:293-316.

20 New DAT. Whole-embryo culture and the study of mammalian embryos during organogenesis. Biol Rev 1978;53:81-122.

21 Cockroft DL. Dissection and culture of postimplantation embryos. In: Copp AJ, Cockroft DL, eds. Postimplantation mammalian embryos: a practical approach. Oxford: IRL Press, 1990:15-40.

22 Evans EP, Burtenshaw MD, Ford CE. Chromosomes of mouse embryos and newborn young: preparations from mouse embryos and newborn young: preparations from

23 Bailey NT. Statistical methods in biology. London: Edward Arnold, 1981 .

24 Tsunoda Y, Tokunaga T, Sugie T. Altered sex ratio of live young after transfer of fast- and slow-developing mouse ;12:301-4.

25 Gardner DK, Leese HJ. Assessment of embryo viability prior to transfer by the non-invasive measurement of glucose uptake. $f$ Exp Zool 1987;242:103-5.

26 Avery B, Madison V, Greve R. Sex and development in bovine in vitro fertilised embryos. Theriogenology 191.35.953-63.

27 Xu KP, Yadav BR, King WA, Betteridge KJ. Sex-related differences in developmental rates of bovine embryos produced and cultured in vitro. Mol Reprod Dev 1992;31:249-5

28 Epstein CJ, Smith S, Travis B, Tucker G. Both X chromosomes function before visible $\mathrm{X}$-chromosome inactivation in female mouse embryos. Nature 1978;274:500-3.

29 Thornhill AR, Burgoyne PS. A paternally imprinted X chromosome retards the development of the early mouse embryo. Development 1993;118:171-4.

30 Burgoyne PS. A Y-chromosomal effect on blastocyst cel number in mice. Development 1993;117:341-5.

31 Webster WS, Edwards MJ. Hyperthermia and the induction of neural tube defects in mice. Teratology 1984;29:417-25.

32 Sadler TW, New DAT. Culture of mouse embryos during neurulation. f Embryol Exp Morphol 1981;66:109-16.

33 Adinolfi M, Beck SE, Embury S, Polani PE, Seller MJ. Levels of alpha-fetoprotein in amniotic fluids of mice (curly tail) with neural tube defects. I Med Genet 1976;13:511-13.

34 Neumann PE, Frankel WN, Letts VA, Coffin JM, Copp AJ, Bernfield M. Multifactorial inheritance of neural tube defects: localization of the major gene and recognition of modifiers in $c t$ mutant mice. Nature Genet (in press). 